\title{
Predictors of complications after endoscopic retrograde cholangiopancreatography: a prognostic model for early discharge
}

\author{
S. M. Jeurnink $\cdot$ P. D. Siersema $\cdot$ E. W. Steyerberg $\cdot$ \\ J. Dees $\cdot$ J. W. Poley $\cdot$ J. Haringsma $\cdot$ E. J. Kuipers
}

Received: 25 July 2010/Accepted: 1 February 2011/Published online: 1 April 2011

(C) The Author(s) 2011. This article is published with open access at Springerlink.com

\begin{abstract}
Background Several studies have evaluated predictors for complications of endoscopic retrograde cholangiopancreatography (ERCP), but their relative importance is unknown. In addition, currently used blood tests to detect post-ERCP pancreatitis are inconsistent. The aim of this study was to determine predictors of post-ERCP complications that could discriminate between patients at highest and lowest risk of post-ERCP complications and to develop a model that is able to identify patients that can safely be discharged shortly after ERCP.

Methods In a single-center, retrospective analysis over the period 2002-2007, predictors of post-ERCP complications were evaluated in a multivariable analysis and compared with those identified from a literature review. A prognostic model was developed based on these risk factors, which was further evaluated in a prospective patient population.
\end{abstract}

S. M. Jeurnink · P. D. Siersema $\cdot$ J. Dees

J. W. Poley · J. Haringsma · E. J. Kuipers

Department of Gastroenterology and Hepatology, Erasmus

MC/University Medical Center Rotterdam, Rotterdam,

The Netherlands

E. W. Steyerberg

Department of Public Health, Erasmus MC/University Medical

Center Rotterdam, Rotterdam, The Netherlands

S. M. Jeurnink · P. D. Siersema

Department of Gastroenterology and Hepatology, University

Medical Center Utrecht, Utrecht, The Netherlands

S. M. Jeurnink $(\square)$

Department of Gastroenterology and Hepatology,

University Medical Center Utrecht, Heidelberglaan 100, 3584,

CX, Utrecht, The Netherlands

e-mail: s.m.jeurnink@students.uu.nl
Results From our retrospective analysis and literature review, we selected the eight most important risk factors for post-ERCP pancreatitis and cholangitis. In the prognostic model, the risk factors (precut) sphincterotomy, sphincter of Oddi dysfunction, younger age, female gender, history of pancreatitis, pancreas divisum, and difficult cannulation accounted for a score of 1 each, whereas primary sclerosing cholangitis (PSC) accounted for a score of 2. A sum score of 4 or more in the prognostic model was associated with a high risk of developing pancreatitis and cholangitis $(27 \% ; 6 / 22)$ in the prospective patient population, whereas a sum score of 3 or less was associated with a low to intermediate risk $(8 \% ; 20 / 252)$.

Conclusions We identified specific patient- and procedure-related factors that are associated with post-ERCP complications. The prognostic model based on these factors is able to identify patients who can be safely discharged the same day after ERCP.

Keywords ERCP - Complications - Risk factors · Early discharge

Endoscopic retrograde cholangiopancreatography (ERCP) is a commonly performed procedure for the diagnosis and treatment of biliary and pancreatic duct disorders. However, ERCP is associated with a relatively high complication rate. In published series, these rates vary between 0.8 and $45 \%$, with pancreatitis occurring in $1-5 \%$, cholangitis in $1-5 \%$, retroperitoneal perforation in $1-2 \%$, and hemorrhage in $1 \%$ [1-7].

Several retrospective and prospective trials have evaluated risk factors for post-ERCP complications. The relative contribution of risk factors to morbidity and mortality after ERCP, however, is unknown. Identification of these risk 
factors can, for example, be used to recognize patients at highest risk for post-ERCP complications, for whom preventive endoscopic or pharmacologic measures and postprocedural monitoring should be considered. Risk factors may also aid in distinguishing patients at lowest risk for complications as they are eligible for undergoing ERCP as an outpatient procedure [1, 3-5, 8-14]. Early discharge can lead to a decrease in the burden of ERCP for patients and an overall cost reduction. So far, only Friedland et al. [14] has presented a prognostic model that was able to predict the risk of post-ERCP pancreatitis. However, this model did not consider other common ERCP complications such as cholangitis, hemorrhage, and perforation. In addition, current blood tests, such as amylase and lipase levels for pancreatitis, are inconsistent and do not aid sufficiently in clinical decision-making on whether patients can safely be discharged early after ERCP $[15,16]$.

The objective of this study was to examine risk factors for post-ERCP complications, i.e., pancreatitis and cholangitis, that could aid clinicians in identifying high- and low-risk patient groups for post-ERCP complications. We compared the risk factors from a multivariable analysis in a retrospectively collected single-center patient population with those obtained from a literature review. In order to determine which patients could safely be discharged after ERCP, we developed a prognostic model for post-ERCP pancreatitis and cholangitis and evaluated this in a prospective patient population.

\section{Materials and methods}

\section{Patients}

For the retrospective analysis, we included all patients who underwent ERCP in our center between January 2002 and January 2007. For the prospective analysis, we included all consecutive patients who underwent ERCP between April 2007 and April 2009. All data on patient and treatment characteristics, complications, and mortality after ERCP were collected. For the prospective analysis, complications after discharge were evaluated by telephone interviews with patients at 7 and 30 days after ERCP and by diaries for pain and nausea scores. Patients who were referred from other medical centers only for ERCP examination were excluded from the analysis because of uncertainty with regard to the completeness of the 30 day follow-up data according to our strict protocol.

\section{Procedure}

The study setting was an academic tertiary referral center with a liver transplant program and an ERCP volume averaging between 650 and 800 ERCPs per year over the last 10 years. All ERCP procedures were performed by experienced endoscopists, generally assisted by a fellowtrainee. A plain abdominal X-ray was made immediately after the ERCP. All patients were admitted overnight following the procedure.

\section{Systematic review}

A systematic review of the literature was performed by searching PubMed, EMBASE, the Cochrane Library, and the Web of Science for the period January 1985-September 2009, using the following search terms: ERCP, pancreatitis, cholangitis, and hemorrhage. In addition, we checked references of detected studies to identify additional relevant reports. A total of 736 studies were found using these search terms, of which 20 reported a univariable and/or multivariable analysis of risk factors for post-ERCP complications. One study was excluded because the relevant data for calculating the confidence intervals was not reported. Another study was excluded because it was not available in the English language. Finally, a total of 18 studies was included [1, 3-6, 8, 10-14, 17-23].

\section{Definitions}

Procedure-related complications were defined as any event occurring during the 30 day period after ERCP that negatively affected the health status of patients for any period of time and graded into categories of severity as proposed by Cotton et al. [24]. Complications were defined according to the literature as follows:

- Pancreatitis: increased serum amylase concentration of at least three times the normal level and occurring $24 \mathrm{~h}$ or more after ERCP with concomitant new (or worsening of) abdominal pain.

- Cholangitis: fever due to (sub-) total obstruction of the biliary system which was present for a minimum of $24 \mathrm{~h}$ after ERCP.

- Hemorrhage: bleeding occurring during or shortly after ERCP and associated with a hemoglobin drop of at least $3 \mathrm{~g} / \mathrm{dl}$. Immediate minor hemorrhage was carefully observed, but this was not considered to be a complication.

- Perforation: radiological presence of contrast or air outside the confines of the bile duct and duodenum during or after ERCP and often seen on a plain abdominal X-ray immediately after the procedure.

In patients with active pancreatitis or cholangitis at the time of ERCP, symptoms compatible with these conditions in the postprocedural phase of the study were not scored as procedural complications. Similarly, when progression of 
the underlying disease resulted in death within 30 days after ERCP, this was also considered to be unrelated to the procedure [6]. The time after which complications occurred was defined as the time that the first signs of pancreatitis, cholangitis, hemorrhage, or perforation became clinically evident.

\section{Statistics}

For the analysis of the retrospective series, explanatory risk factors for complications identified in the reviewed literature were applied. We did not account for multiple procedures in the same patient. Logistic regression was performed using Statistical Package for Social Science program (SPSS 12.0.1, SPSS Inc., Chicago, IL). Factors that had a $P<0.5$ in univariable analysis were entered into a stepwise logistic regression model to estimate adjusted odds ratios (ORs). In the multivariable analysis, a $P<0.05$ was considered to be significant.

Forest plots were constructed using ORs and 95\% confidence intervals (CIs) of risk factors for complications when these were evaluated in more than two reviewed studies. ORs were pooled and forest plots were created using StatsDirect (StatsDirect statistical tools, StatsDirect Ltd., UK). A $Z$ test was used to test whether pooled ORs differed from 1 , indicating a significant effect on the development of post-ERCP complications. A two-sided $P<0.05$ was considered to be statistically significant.

Two experienced gastroenterologists, both performing over 200 ERCPs annually, selected the most important risk factors from the retrospective analysis and literature review for post-ERCP pancreatitis and cholangitis to be included in a prognostic model. Each risk factor in the prognostic model was nominated a value that represented the magnitude of the effect on the development of pancreatitis or cholangitis, based on the typical ORs. Risk factors were evaluated using logistic regression in the prospective patient population using SPSS software ver. 12.0.1. A Kaplan-Meier curve was used to determine the time to complications. A two-sided $P<0.05$ was considered statistically significant.

\section{Results}

Retrospective patient population

A total of 588 patients $(58 \%$ male, mean age $=56.5 \pm$ 17 years), who underwent a total of 1,372 ERCPs, were included in this part of the study. Characteristics of the ERCP procedures are presented in Table 1. A total of $76(6 \%)$ complications occurred after ERCP, of which pancreatitis
Table 1 Characteristics of the ERCP procedure in a retrospective patient population

\begin{tabular}{ll}
\hline Characteristics & $N$ \\
\hline Number of procedures & 1,372 \\
Number of patients & 588 \\
Diagnostic procedures & $199(15 \%)$ \\
Failed procedures & $130(10 \%)$ \\
First time ERCPs & $367(27 \%)$ \\
Procedures & \\
Sphincterotomy & $225(16 \%)$ \\
Precut sphincterotomy & $74(5 \%)$ \\
Plastic endoprosthesis placement & $702(51 \%)$ \\
Metal stent placement & $52(4 \%)$ \\
Balloon dilation of CBD & $130(9 \%)$ \\
Removal of CBD stones & $186(14 \%)$ \\
Ampullary resection & $8(1 \%)$ \\
\hline$C B D$ comm
\end{tabular}

$C B D$ common bile duct

was seen in $34(2 \%)$ procedures, cholangitis in $31(2 \%)$, perforation in $6(0.4 \%)$, and hemorrhage in $5(0.4 \%)$.

Uni- and multivariable analyses of the retrospective population and systematic review

We found 12 risk factors that were significantly associated with overall post-ERCP complications in our patient population in the univariable analysis (Table 2). In addition, 11 patient- and procedure-related risk factors for post-ERCP pancreatitis (Table 3) and ten for cholangitis were identified (Table 4).

In the multivariable analysis, only primary sclerosing cholangitis (PSC) (OR 2.2, CI 1.1-4.4) and sphincterotomy (OR 2.2, CI 1.3-3.9) remained statistically significant for overall post-ERCP complications. Pooled ORs from the systematic review resulted in three statistically significant risk factors for overall post-ERCP complications, i.e., suspected sphincter of Oddi dysfunction (SOD) (OR 2.6, CI 2.0-3.3), precut sphincterotomy (OR 1.7, CI 1.5-2.0), and female gender (OR 1.4, CI 1.2-1.5).

Significant risk factors for post-ERCP pancreatitis in the multivariable analysis included pancreas divisum (OR 10.5, CI 1.0-112.8), PSC (OR 4.6, CI 1.8-11.5), age $<60$ years (OR 4.9, CI: 1.2-19.6), and female gender (OR 2.1, CI 1.0-4.6). Pooled ORs from the systematic review resulted in eight statistically significant risk factors for post-ERCP pancreatitis, i.e., suspected SOD (OR 3.6, CI 2.3-5.3), history of post-ERCP pancreatitis (OR 1.9, CI 1.6-2.4), difficult cannulation (OR 2.5, CI 2.0-3.2), precut sphincterotomy (OR 2.4, CI 1.8-3.2), pancreas divisum (OR 2.2, CI 1.4-3.4), younger age (OR 2.0, CI 1.6-2.1), 
Table 2 Pooled odds ratios of predictors for overall post-ERCP complications from the literature review and odds ratios from univariable and multivariable analyses of a retrospectively collected database

\begin{tabular}{|c|c|c|c|}
\hline \multirow[t]{2}{*}{ Predictors } & \multirow[t]{2}{*}{ Literature review } & \multicolumn{2}{|c|}{ Retrospective population } \\
\hline & & Univariable analysis & Multivariable analysis \\
\hline \multicolumn{4}{|l|}{ Patient variables } \\
\hline Suspected SOD & $2.6(2.0-3.3) \dagger$ & NA & NA \\
\hline Female gender & $1.4(1.2-1.5) \dagger$ & $1.6(0.9-2.2) \dagger$ & $1.3(0.8-2.1)$ \\
\hline History of pancreatitis & NA & $0.9(0.5-1.7)$ & NS \\
\hline Younger age ( $<60$ years $)$ & $1.0(1.0-1.1)$ & $1.3(0.8-2.2) \dagger$ & $1.7(0.7-3.8)$ \\
\hline Age (continuous) & NA & $1.0(1.0-1.02) \dagger$ & $1.0(1.0-1.0)$ \\
\hline Cholangitis at presentation & NA & $0.4(0.2-1.0) \dagger$ & $0.5(0.2-1.2)$ \\
\hline Antibiotic use & NA & $0.7(0.4-1.5) \dagger$ & $0.8(0.4-1.6)$ \\
\hline Cirrhosis & NA & $1.3(0.4-3.6)$ & NS \\
\hline PSC & NA & $1.8(1.0-3.3) \dagger$ & $2.2(1.1-4.4) \dagger$ \\
\hline Previous ERCP & NA & $0.7(0.4-1.2) \dagger$ & $0.9(0.5-1.7)$ \\
\hline \multicolumn{4}{|l|}{ Treatment variables } \\
\hline Therapeutic ERCP & NA & $1.7(0.8-3.8) \dagger$ & $1.4(0.6-3.1)$ \\
\hline Precut sphincterotomy & $1.7(1.5-2.0) \dagger$ & $1.9(0.8-4.2) \dagger$ & $1.6(0.6-4.2)$ \\
\hline Sphincterotomy & NA & $2.3(1.4-3.9) \dagger$ & $2.2(1.3-3.9) \dagger$ \\
\hline Balloon dilation CBD & NA & $1.5(0.7-3.0) \dagger$ & $1.6(0.8-3.3)$ \\
\hline Difficult cannulation & NA & $1.6(0.9-2.7) \dagger$ & $1.3(0.7-2.4)$ \\
\hline
\end{tabular}

Values are odds ratio (confidence interval)

$S O D$ sphincter of Oddi dysfunction, $P S C$ primary sclerosing cholangitis, $C B D$ common bile duct, $N A$ not applicable, $N S$ not significant

$\dagger P<0.5$ in univariable analysis and $P<0.05$ in multivariable analysis

female gender (OR 1.6, CI 1.3-1.8), and multiple pancreatic duct contrast injections (OR 1.6, CI 1.3-2.0).

In the multivariable analysis for post-ERCP cholangitis, significant risk factors were self-expanding metal stent (SEMS) placement (OR 3.9, CI 1.0-15.7) and sphincterotomy (OR 2.8, CI: 1.2-6.4). Pooled ORs from the systematic review resulted in four statistically significant risk factors for post-ERCP cholangitis, i.e., jaundice at presentation (OR 4.8, CI 1.6-14.3), a small-volume center (OR 4.7, CI 1.9-11.7), plastic stent placement (OR 3.1, CI 1.8-5.2), and female gender (OR 2.8, CI 1.2-6.6).

Prospective patient population

A total of 1,478 ERCPs were performed between April 2007 and April 2009, of which 951 ERCPs were performed in patients referred from another hospital and therefore were excluded. In total, we included 274 ERCPs (253 ERCPs were performed in patients who refused to participate in follow-up), which were performed in 220 patients (59\% male, mean age $=60 \pm 14$ years), in the prospective study (Table 5). In 10\% of the ERCP procedures (27/274), post-ERCP complications developed during a 30 day follow-up, i.e., 14 episodes of pancreatitis (5\%), 12 of cholangitis $(5 \%)$, and 1 hemorrhage $(0.1 \%)$. Pancreatitis severity was mild after three, moderate after eight, and severe after three ERCPs. Cholangitis severity was mild after three and moderate after nine ERCPs. The case of hemorrhage was graded as mild. None of the patients had more than one complication.

Time to complication

Post-ERCP complications, i.e., pancreatitis and cholangitis, occurred after a mean time of $4.1 \mathrm{~h}$ after ERCP, subdivided in $4.2 \mathrm{~h}$ for pancreatitis and $4.1 \mathrm{~h}$ for cholangitis. Overall, $90 \%(23 / 26)$ of complications were detected within $6 \mathrm{~h}$ after treatment. Complications occurring more than $6 \mathrm{~h}$ after ERCP were cholangitis in 2 (after 7 and $16 \mathrm{~h}$ ) and pancreatitis in 1 (after $17 \mathrm{~h}$ ) (Fig. 1).

Prognostic model

The prognostic model for post-ERCP pancreatitis and cholangitis included eight risk factors. All these factors were evaluated in the prospective patient series by multivariable logistic regression. Risk factors included (precut) sphincterotomy (OR 1.6, CI 0.6-4.0), suspected SOD (not defined), younger age ( $<60$ years) (OR 1.0, CI 0.4-2.4), PSC (OR 1.9, CI 0.5-8.0), female gender (OR 0.6, CI $0.2-1.5$ ), history of pancreatitis (OR 1.4, CI 0.5-3.7), pancreas divisum (OR 2.6, CI 0.2-30.5), and difficult 
Table 3 Pooled odds ratios of predictors for post-ERCP pancreatitis from the literature review and odds ratios from univariable and multivariable analyses of a retrospectively collected database

\begin{tabular}{|c|c|c|c|}
\hline \multirow[t]{2}{*}{ Predictors } & \multirow[t]{2}{*}{ Literature review } & \multicolumn{2}{|c|}{ Retrospective population } \\
\hline & & Univariate analysis & Multivariable analysis \\
\hline \multicolumn{4}{|l|}{ Patient variables } \\
\hline Younger age ( $<60$ years $)$ & $2.0(1.6-2.5) \dagger$ & $4.0(1.5-10.5) \dagger$ & $4.9(1.2-19.6) \dagger$ \\
\hline Age (continuous) & NA & $1.0(1.0-1.1) \dagger$ & $1.0(1.0-1.0)$ \\
\hline Female gender & $1.6(1.3-1.8) \dagger$ & $1.8(0.9-3.5) \dagger$ & $2.1(1.0-4.6) \dagger$ \\
\hline History of pancreatitis & $1.9(1.6-2.4) \dagger$ & $1.4(0.6-3.2) \dagger$ & $1.8(0.7-4.7)$ \\
\hline PSC & NA & $3.4(1.6-7.2) \dagger$ & $4.6(1.8-11.5) \dagger$ \\
\hline Suspected SOD & $3.6(2.3-5.3) \dagger$ & NA & NA \\
\hline Small-diameter CBD & $1.5 \mathrm{NS}$ & NA & NA \\
\hline Pancreas divisum & $2.2(1.4-3.4) \dagger$ & $6.7(0.8-57.5) \dagger$ & $10.5(1.0-112.8) \dagger$ \\
\hline Cholangitis at presentation & NA & $0.2(0.0-1.3) \dagger$ & $0.2(0.03-1.6)$ \\
\hline Previous ERCP & NA & $0.6(0.3-1.2) \dagger$ & $0.6(0.2-1.3)$ \\
\hline \multicolumn{4}{|l|}{ Treatment variables } \\
\hline Precut sphincterotomy & $2.4(1.8-3.2) \dagger$ & $1.1(0.3-4.7)$ & $1.3(0.3-6.1)$ \\
\hline Multiple PD contrast injections & $1.6(1.3-2.0) \dagger$ & NA & NA \\
\hline Pancreatic sphincterotomy & $1.2(0.9-1.4)$ & NA & NA \\
\hline Therapeutic ERCP & NA & $1.3(0.5-3.7)$ & NS \\
\hline Sphincterotomy & NA & $1.9(0.9-4.1) \dagger$ & $1.5(0.5-4.0)$ \\
\hline Placement of endoprothese & NA & $0.8(0.4-1.7)$ & NS \\
\hline Balloon dilation CBD & NA & $2.1(0.9-5.2) \dagger$ & $2.2(0.8-5.8)$ \\
\hline Stone removal & NA & $2.0(0.9-4.5) \dagger$ & $1.9(0.7-4.9)$ \\
\hline Difficult cannulation & $2.5(2.0-3.2) \dagger$ & $1.1(0.5-2.6)$ & NS \\
\hline
\end{tabular}

Values are odds ratio (confidence interval)

$S O D$ sphincter of Oddi dysfunction, $P S C$ primary sclerosing cholangitis, $C B D$ common bile duct, $N A$ not applicable, $N S$ not significant, $P D$ pancreatic duct

$\dagger P<0.5$ in univariable analysis and $P<0.05$ in multivariable analysis

cannulation ( $>10 \mathrm{~min}$ attempting to cannulate) (OR 2.0, CI 0.7-5.6). The sum score for each of the risk factors was derived from the coefficients of the regression analysis from the review (Table 6). For example, a 58-year-old female patient with PSC and undergoing an ERCP with precut sphincterotomy has a sum score of $5(1+1+$ $2+1)$

Patients were scored as having a high risk (overall sum score $>3$ ) or a low to intermediate risk (overall sum score $\leq 3$ ). In the low- to intermediate-risk patient group, complications occurred in $8 \%(20 / 252)$. In the high-risk group, complications occurred in $27 \%(6 / 22)$ (Table 7$)$.

\section{Discussion}

We present an overview of prognostic factors for (specific) post-ERCP complications. Our prognostic model based on these risk factors was able to distinguish between patients at a high or a low risk of developing post-ERCP complications. This model may aid in the decision of whether patients can be safely discharged at an early stage after ERCP.

The data obtained from our systematic literature review and the multivariable analysis of a large retrospective population resulted in an accurate prediction model. This model was based on a more or less complete overview of all possible risk factors for post-ERCP pancreatitis and cholangitis. Nevertheless, we recognize that using these data may have some limitations. The risk factors in the systematic review were derived from several studies and therefore risk factors from heterogeneous patient groups may have been included. For example, in some studies only patients undergoing sphincterotomy or patients with SOD were evaluated [4, 12, 19].

As expected, the results from the systematic review of the literature and our retrospectively evaluated patient population partially overlapped. Nevertheless, there were some differences. For example, female gender was significant only in the univariable analysis of our population, whereas it was a significant risk factor according to pooled ORs $[5,14,17,19,20]$. Several studies have suggested that 
Table 4 Pooled odds ratios of predictors for post-ERCP cholangitis from the literature review and odds ratios from univariable and multivariable analyses of a retrospectively collected database

\begin{tabular}{|c|c|c|c|}
\hline \multirow[t]{2}{*}{ Predictors } & \multirow[t]{2}{*}{ Literature review } & \multicolumn{2}{|c|}{ Retrospective population } \\
\hline & & Univariate analysis & Multivariable analysis \\
\hline \multicolumn{4}{|l|}{ Patient variables } \\
\hline Age (continuous) & NA & $1.0(1.0-1.0) \dagger$ & $1.0(1.0-1.0)$ \\
\hline Younger age ( $<60$ years) & NA & $0.6(0.3-1.3) \dagger$ & $0.7(0.2-2.5)$ \\
\hline Female gender & $2.8(1.2-6.6) \dagger$ & $0.7(0.3-1.6) \dagger$ & $0.7(0.3-1.6)$ \\
\hline Small center & $4.7(1.9-11.7) \dagger$ & NA & NA \\
\hline Jaundice at presentation & $4.8(1.6-14.3) \dagger$ & $0.7(0.3-1.8) \dagger$ & $0.6(0.2-1.6)$ \\
\hline Antibiotic use & $0.9 \mathrm{NS}$ & $1.6(0.5-5.3) \dagger$ & $0.7(0.3-2.2)$ \\
\hline Previous ERCP & NA & $1.5(0.6-3.8) \dagger$ & $1.4(0.5-3.8)$ \\
\hline Previous precut & NA & $2.3(0.9-5.6) \dagger$ & $2.2(0.9-5.8)$ \\
\hline \multicolumn{4}{|l|}{ Treatment variables } \\
\hline Obstruction of CBD at end ERCP & $0.3 \mathrm{NS}$ & NA & NA \\
\hline Placement expandable stent & NA & $2.8(0.8-9.6) \dagger$ & $3.9(1.0-15.7) \dagger$ \\
\hline Difficult cannulation & NA & $1.2(0.5-2.9)$ & NS \\
\hline Sphincterotomy & NA & $2.1(1.0-4.7) \dagger$ & $2.8(1.2-6.4) \dagger$ \\
\hline Precut sphincterotomy & NA & $1.2(0.3-5.2)$ & NS \\
\hline Placement of endoprothesis & $3.1(1.8-5.2)$ & $1.3(0.6-2.7) \dagger$ & $1.8(0.8-3.9)$ \\
\hline
\end{tabular}

Values are odds ratio (confidence interval)

$C B D$ common bile duct, $N A$ not applicable, $N S$ not significant in univariable analysis

$\dagger P<0.5$ in univariable analysis and $P<0.05$ in multivariable analysis

Table 5 Characteristics of the ERCP procedure in a prospective patient population

\begin{tabular}{ll}
\hline Characteristics & Number $(\%)$ \\
\hline Number of procedures & 274 \\
Number of patients & 220 \\
Gender (\% male) & $129(59)$ \\
Age ( \pm SD) & $60( \pm 14)$ \\
Diagnostic procedures (\%) & $59(22)$ \\
Virgin ERCPs (\%) & $77(28)$ \\
Indication & \\
CBD stones & 62 \\
Stenosis of the anastomosis after LTx & 42 \\
Malignant CBD obstruction & 29 \\
Chronic pancreatitis & 28 \\
PSC & 21 \\
Acute pancreatitis & 11 \\
PBC & 2 \\
Other & 79 \\
Procedures & \\
(Precut) sphincterotomy & 67 \\
Placement of a plastic endoprothesis/stent & 149 \\
Balloon dilation of the CBD & 41 \\
Removal of CBD stones & 46 \\
Ampullary resection & 7 \\
\hline CBD comm bile & \\
\hline
\end{tabular}

$C B D$ common bile duct, $L T x$ liver transplant, PSC primary sclerosing cholangitis women are at a higher risk of post-ERCP complications, which can be explained by the higher prevalence of SOD, regardless of the clinical context or technical difficulty of the ERCP [4, 10, 13]. The prevalence of (recognized) SOD was low in our ERCP population and this may explain the difference in the effect of gender with findings in the literature. For these reasons, we included female gender in the prognostic model for post-ERCP complications.

Precut sphincterotomy has also been recognized as a risk factor for post-ERCP complications. In published series

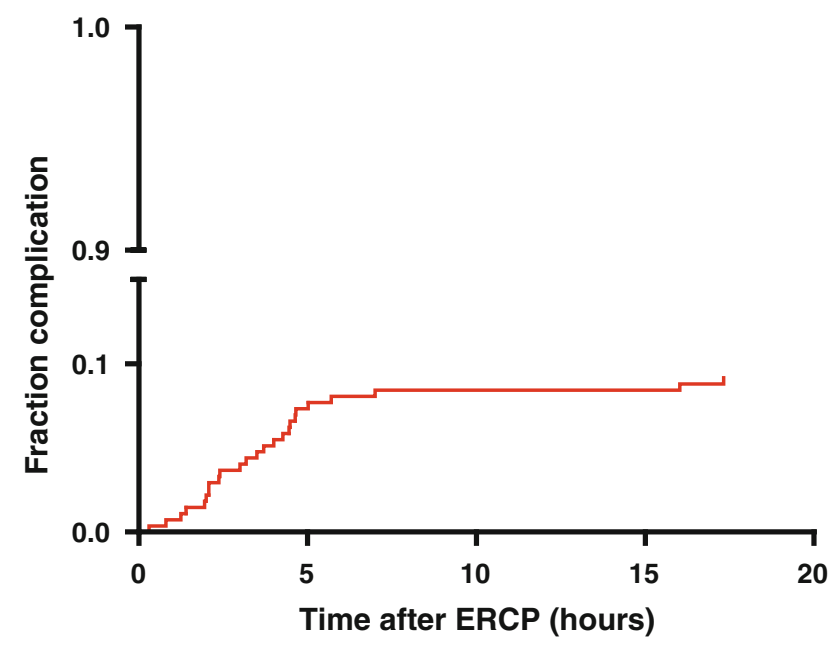

Fig. 1 Time between ERCP and complication 
this risk factor is suggested to be largely dependent on experience of an endoscopist [3, 4, 17]. Nevertheless, we were not able to establish precut sphincterotomy as a risk factor in our series. This is likely to be due to the fact that precut sphincterotomy was performed only by highly experienced endoscopists in our center. Precut sphincterotomy in itself is often associated with a difficult cannulation. As the latter was not a recorded risk factor for ERCP complications in our retrospective database, precut sphincterotomy might well be a substitute for this complication, explaining the difference with literature findings. Again, for these reasons, we included (precut) sphincterotomy as well as difficult cannulation in the prognostic model for post-ERCP complications.

A history of post-ERCP pancreatitis has also been reported to be a risk factor for post-ERCP pancreatitis $[1,12,14]$. However, this was also not the case in our population. We suspect that a history of pancreatitis is not a

Table 6 Combined scores for a prognostic model of pancreatitis and cholangitis and ORs from the literature and a multivariable analysis of a retrospective $(n=1372)$ and prospective $(n=255)$ patient population

\begin{tabular}{lc}
\hline Characteristics & Score \\
\hline (Precut) sphincterotomy & +1 \\
SOD & +1 \\
Younger age $(<60)$ & +1 \\
PSC & +2 \\
Female gender & +1 \\
History of pancreatitis & +1 \\
Pancreas divisum $^{\text {Difficult cannulation }}{ }^{\mathrm{a}}$ & +1 \\
\hline
\end{tabular}

$S O D$ sphincter of Oddi dysfunction, PSC primary sclerosing cholangitis

${ }^{\text {a }}$ Defined as more than 10 min of attempting to cannulate

Table 7 Post-ERCP pancreatitis or cholangitis by score

\begin{tabular}{llll}
\hline $\begin{array}{l}\text { Score } \\
\text { ERCPs without } \\
\text { cholangitis or } \\
\text { pancreatitis }\end{array}$ & $\begin{array}{l}\text { ERCPs with } \\
\text { cholangitis or } \\
\text { pancreatitis (\%) }\end{array}$ & Risk groups \\
\hline 0 & 38 & $3(7)$ & $\begin{array}{l}\text { Low- to intermediate-risk } \\
\text { group: }\end{array}$ \\
1 & 84 & $11(12)$ & $\begin{array}{l}252 \text { patients (92\% of total) } \\
8 \% \text { risk }\end{array}$ \\
2 & 70 & $5(6)$ & High-risk group: \\
3 & 40 & $1(7)$ & 22 patients (8\% of total) \\
\hline 4 & 15 & $5(25)$ & $27 \%$ risk \\
$5+$ & 1 & $1(50)$ & \\
\hline
\end{tabular}

strong risk factor for recurrent pancreatitis, as it was found to be significant only in univariable analysis in the literature review [1, 12, 14].

The multivariable analysis of risk factors in our retrospective population established some factors that were not previously evaluated in the literature. For example, we found that the presence of PSC was a risk factor for postERCP pancreatitis. Previous studies have suggested that this is a risk factor for post-ERCP complications due to the often difficult and multiple cannulation attempts in PSC patients, particularly in the presence of complicated strictures $[25,26]$. In addition, we also found that younger age was a risk factor for pancreatitis. Freeman et al. [13] have suggested that younger age as a risk factor was in fact caused by the fact that authors did not correct for confounding variables. An explanation for younger age as risk factor may also be the progressive decline in pancreatic exocrine function that occurs with aging, which may protect older patients from developing pancreatitis as a consequence of ERCP.

We excluded all patients who were referred from other hospitals, and investigated only patients who were primarily admitted to our university hospital. The latter group is likely to have more comorbidity compared to patients treated in regional hospitals, which could have influenced our results.

Our prognostic model was able to distinguish patients with a low to intermediate risk from those with a high risk for post-ERCP pancreatitis or cholangitis, resulting in $8 \%$ of all patients being at high risk. These results are comparable to the results of the prognostic model developed by Friedland et al. [14], who found that $7 \%$ of all patients undergoing ERCP were at a high risk for postERCP pancreatitis. Friedland et al. [14] were the first to propose a simple prognostic model for post-ERCP pancreatitis composed of the risk factors of pain during the procedure, cannulation of the pancreatic duct, history of pancreatitis, and difficult cannulation. Their model was based on a multivariable analysis of retrospectively collected data and therefore missed various risk factors known from other studies, i.e., SOD, gender, and precut sphincterotomy. In addition, the model was validated using the same database that was used to determine the risk factors for the prognostic model. Evaluation of a prognostic model in another population is preferable. This should enable one to determine whether the model is indeed generally applicable.

Besides the limitations from the systematic review and the retrospective patient population, our prognostic model has additional limitations. When we compared the selected risk factors in our prospective patient population with the pooled ORs from the systematic review, we found differences in the level of significance, probably a result of the 
Fig. 2 Proposal of a decision model for early discharge after ERCP

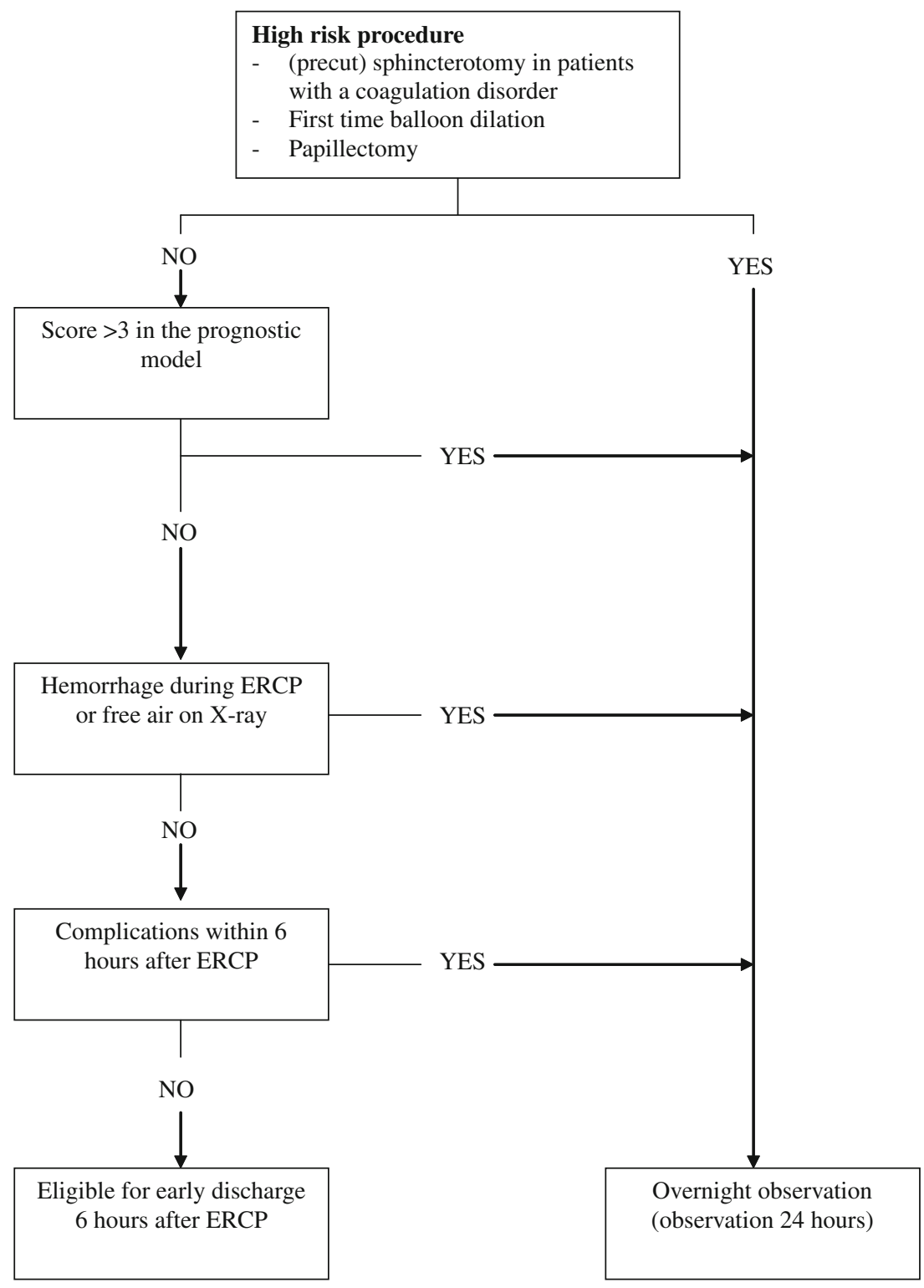

relatively small study population. Nonetheless, the magnitude of the ORs of the risk factors was comparable.

A few studies have suggested that size of a center and experience of an endoscopist both have an effect on the risk of post-ERCP complications. Therefore, our model can be used only in patients undergoing ERCP in a center in which endoscopists perform more than 150 ERCPs per year and also an otherwise experienced team is present [17]. This is illustrated by the findings of Loperfido et al. [17] who reported an increased risk of developing post-ERCP complications in a small center compared to a large center. According to their study, one could correct for this by adding an additional score of 1 to the model in case an ERCP is performed in a small-volume center and/or by a less experienced team. This needs to be investigated in future studies.
In conclusion, our results show that the prognostic model that we developed is able to identify patients with a high risk of developing pancreatitis or cholangitis after ERCP (Fig. 2). On the other hand, it also identifies patients at low to intermediate risk who can safely be discharged $6 \mathrm{~h}$ after ERCP. In addition, we also suggest that patients be admitted overnight when the ERCP is associated with an increased risk of perforation and hemorrhage.

Disclosure S. M. Jeurnink, P. D. Siersema, E. W. Steyerberg, J. Dees, J. W. Poley, J. Haringsma, and E. J. Kuipers have no conflicts of interest or financial ties to disclose.

Open Access This article is distributed under the terms of the Creative Commons Attribution Noncommercial License which permits any noncommercial use, distribution, and reproduction in any medium, provided the original author(s) and source are credited. 


\section{References}

1. Vandervoort J, Soetikno RM, Tham TC, Wong RC, Ferrari AP Jr, Montes H, Roston AD, Slivka A, Lichtenstein DR, Ruymann FW, Van Dam J, Hughes M, Carr-Locke DL (2002) Risk factors for complications after performance of ERCP. Gastrointest Endosc 56:652-656

2. Mehta SN, Pavone E, Barkun JS, Bouchard S, Barkun AN (1998) Predictors of post-ERCP complications in patients with suspected choledocholithiasis. Endoscopy 30:457-463

3. Cheng CL, Sherman S, Watkins JL, Barnett J, Freeman M, Geenen J, Ryan M, Parker H, Frakes JT, Fogel EL, Silverman WB, Dua KS, Aliperti G, Yakshe P, Uzer M, Jones W, Goff J, Lazzell-Pannell L, Rashdan A, Temkit M, Lehman GA (2006) Risk factors for post-ERCP pancreatitis: a prospective multicenter study. Am J Gastroenterol 101:139-147

4. Rabenstein T, Schneider HT, Bulling D, Nicklas M, Katalinic A, Hahn EG, Martus P, Ell C (2000) Analysis of the risk factors associated with endoscopic sphincterotomy techniques: preliminary results of a prospective study, with emphasis on the reduced risk of acute pancreatitis with low-dose anticoagulation treatment. Endoscopy 32:10-19

5. Christoforidis E, Goulimaris I, Kanellos I, Tsalis K, Demetriades C, Betsis D (2002) Post-ERCP pancreatitis and hyperamylasemia: patient-related and operative risk factors. Endoscopy 34:286-292

6. Williams EJ, Taylor S, Fairclough P, Hamlyn A, Logan RF, Martin D, Riley SA, Veitch P, Wilkinson ML, Williamson PR, Lombard M (2007) Risk factors for complication following ERCP: results of a large-scale, prospective multicenter study. Endoscopy 39:793-801

7. Freeman ML (2002) Adverse outcomes of ERCP. Gastrointest Endosc 56:S273-S282

8. Hookey LC, RioTinto R, Delhaye M, Baize M, Le Moine O, Devière J (2006) Risk factors for pancreatitis after pancreatic sphincterotomy: a review of 572 cases. Endoscopy 38:670-676

9. Boender J, Nix GA, de Ridder MA, van Blankenstein M, Schütte HE, Dees J, Wilson JH (1994) Endoscopic papillotomy for common bile duct stones: factors influencing the complication rate. Endoscopy 26:209-216

10. Christensen M, Matzen P, Schulze S, Rosenberg J (2004) Complications of ERCP: a prospective study. Gastrointest Endosc 60:721-731

11. Masci E, Toti G, Mariani A, Curioni S, Lomazzi A, Dinelli M, Minoli G, Crosta C, Comin U, Fertitta A, Prada A, Passoni GR, Testoni PA (2001) Complications of diagnostic and therapeutic ERCP: a prospective multicenter study. Am J Gastroenterol 96:417-423

12. Freeman ML, Nelson DB, Sherman S, Haber GB, Herman ME, Dorsher PJ, Moore JP, Fennerty MB, Ryan ME, Shaw MJ, Lande JD, Pheley AM (1996) Complications of endoscopic biliary sphincterotomy. N Engl J Med 335:909-918

13. Freeman ML, DiSario JA, Nelson DB, Fennerty MB, Lee JG, Bjorkman DJ, Overby CS, Aas J, Ryan ME, Bochna GS,
Shaw MJ, Snady HW, Erickson RV, Moore JP, Roel JP (2001) Risk factors for post-ERCP pancreatitis: a prospective, multicenter study. Gastrointest Endosc 54:425-434

14. Friedland S, Soetikno RM, Vandervoort J, Montes H, Tham T, Carr-Locke DL (2002) Bedside scoring system to predict the risk of developing pancreatitis following ERCP. Endoscopy 34: 483-488

15. Abdel Aziz AM, Lehman GA (2007) Pancreatitis after endoscopic retrograde cholangio-pancreatography. World J Gastroenterol 13:2655-2668

16. Artifon EL, Chu A, Freeman M, Sakai P, Usmani A, Kumar A (2010) A comparison of the consensus and clinical definitions of pancreatitis with a proposal to redefine post-endoscopic retrograde cholangiopancreatography pancreatitis. Pancreas 39:530-535

17. Loperfido S, Angelini G, Benedetti G, Chilovi F, Costan F, De Berardinis F, De Bernardin M, Ederle A, Fina P, Fratton A (1998) Major early complications from diagnostic and therapeutic ERCP: a prospective multicenter study. Gastrointest Endosc 48:1-10

18. Deans GT, Sedman P, Martin DF, Royston CM, Leow CK, Thomas WE, Brough WA (1997) Are complications of endoscopic sphincterotomy age related? Gut 41:545-548

19. Barthet M, Lesavre N, Desjeux A, Gasmi M, Berthezene P, Berdah S, Viviand X, Grimaud JC (2002) Complications of endoscopic sphincterotomy: results from a single tertiary referral center. Endoscopy 34:991-997

20. Tzovaras G, Shukla P, Kow L, Mounkley D, Wilson T, Toouli J (2000) What are the risks of diagnostic and therapeutic endoscopic retrograde cholangiopancreatography? Aust N Z J Surg 70:778-782

21. Nelson DB, Freeman ML (1994) Major hemorrhage from endoscopic sphincterotomy: risk factor analysis. J Clin Gastroenterol 19:283-287

22. Wang P, Li ZS, Liu F, Ren X, Lu NH, Fan ZN, Huang Q, Zhang X, He LP, Sun WS, Zhao Q, Shi RH, Tian ZB, Li YQ, Li W, Zhi FC (2009) Risk factors for ERCP-related complications: a prospective multicenter study. Am J Gastroenterol 104:31-40

23. Cotton PB, Garrow DA, Gallagher J, Romagnuolo J (2009) Risk factors for complications after ERCP: a multivariate analysis of 11, 497 procedures over 12 years. Gastrointest Endosc 70:80-88

24. Cotton PB, Lehman G, Vennes J, Geenen JE, Russell RC, Meyers WC, Liguory C, Nickl N (1991) Endoscopic sphincterotomy complications and their management: an attempt at consensus. Gastrointest Endosc 37:383-393

25. Etzel JP, Eng SC, Ko CW, Lee SD, Saunders MD, Tung BY, Kimmey MB, Kowdley KV (2007) Complications after ERCP in patients with primary sclerosing cholangitis. Gastrointest Endosc 67(4):643-648

26. Van den Hazel SJ, Wolfhagen EH, van Buuren HR, van de Meeberg PC, Van Leeuwen DJ (2000) Prospective risk assessment of endoscopic retrograde cholangiography in patients with primary sclerosing cholangitis. Dutch PSC Study Group. Endoscopy 32:779-782 